\title{
FACTORIALITY OF VON NEUMANN ALGEBRAS CONNECTED WITH GENERAL COMMUTATION RELATIONS - FINITE DIMENSIONAL CASE
}

\author{
ILONA KRÓLAK \\ Institute of Mathematics, Wrockaw University \\ Pl. Grunwaldzki 2/4, 50-384 Wroctaw, Poland \\ E-mail: ikrol@math.uni.wroc.pl
}

\begin{abstract}
We study a certain class of von Neumann algebras generated by selfadjoint elements $\omega_{i}=a_{i}+a_{i}^{+}$, where $a_{i}, a_{i}^{+}$satisfy the general commutation relations:

$$
a_{i} a_{j}^{+}=\sum_{r, s} t_{j s}^{i r} a_{r}^{+} a_{s}+\delta_{i j} I d .
$$

We assume that the operator $T$ for which the constants $t_{j s}^{i r}$ are matrix coefficients satisfies the braid relation. Such algebras were investigated in $[\mathrm{BSp}]$ and $[\mathrm{K}]$ where the positivity of the Fock representation and factoriality in the case of infinite dimensional underlying space were shown. In this paper we prove that under certain conditions on the number of generators our algebra is a factor. The result was obtained for $q$-commutation relations by P. Śniady [Snia] and recently by E. Ricard $[R]$. The latter proved factoriality without restriction on the dimension, but it cannot be easily generalized to the general commutation relation case. We generalize the result of Śniady and present a simpler proof. Our estimate for the number of generators in case $q>0$ is better than in [Snia].
\end{abstract}

0. Introduction. Let $\mathcal{H}_{R}$ be a real Hilbert space and $\mathcal{H}=\mathcal{H}_{R}+\mathrm{i} \mathcal{H}_{R}$ be its complexification. Denote by $\overline{\mathcal{H}}$ the complex conjugate of $\mathcal{H}$. Let $\bar{T}: \overline{\mathcal{H}} \otimes \mathcal{H} \rightarrow \mathcal{H} \otimes \overline{\mathcal{H}}$ be linear.

Consider the algebra of all tensors over $\mathcal{H}$ and $\overline{\mathcal{H}}$ plus $I d$ with tensor multiplication as a product denoted by $\Upsilon(\mathcal{H}, \overline{\mathcal{H}})$. Define the Wick algebra as the following quotient algebra:

$$
W(T) \simeq \Upsilon(\mathcal{H}, \overline{\mathcal{H}}) /\langle\bar{f} \otimes g-\bar{T}(\bar{f} \otimes g)-\langle g \mid f\rangle I d\rangle
$$

2000 Mathematics Subject Classification: Primary 81S05; Secondary 46L35.

Key words and phrases: general commutation relations, von Neumann algebras, factors.

This paper was partially supported by KBN grant no 2P03A00732 and also by RTN grant HPRN-CT-2002-00279.

The paper is in final form and no version of it will be published elsewhere. 
For $f, g \in \mathcal{H}$ we set $a(f)=\bar{f}, a^{+}(g)=g$, where the action of the operators $a(f)$ and $a^{+}(g)$ is given by multiplication by $\bar{f}$ and $g$, respectively. We are interested in positive representations of $W(T)$, that is, the representations of the $a(f)$ 's as operators on a Hilbert space such that $a^{+}(f)$ is the restriction of the operator adjoint to $a(f)$.

In this paper we will deal with the Fock representation. It is constructed from a cyclic vector $\Omega$ with the property $a(f) \Omega=0$ for all $f \in \mathcal{H}$. Such a representation is unique (up to isomorphism) and carries a unique hermitian form. There are various criteria implying that this form is positive definite. Most of them are written in the language of the properties of the operator $T \in L(\mathcal{H} \otimes \mathcal{H})$ defined as $\langle T(f \otimes g) \mid h \otimes w\rangle=\langle\bar{T}(\bar{h} \otimes f) \mid w \otimes \bar{g}\rangle$. It was proved (see [BSp]) that if $T$ is a selfadjoint contraction and satisfies the braid relation (or Yang-Baxter relation)

$$
[(1 \otimes T)(T \otimes 1)(1 \otimes T)=(T \otimes 1)(1 \otimes T)(T \otimes 1)] \quad \text { on } \mathcal{H} \otimes \mathcal{H} \otimes \mathcal{H}
$$

then the form is positive definite. In such a case the completion of the Fock space

$$
\mathcal{F}^{\text {fin }}(\mathcal{H})=\mathbf{C} \Omega \oplus \bigoplus_{n=1}^{\infty} \mathcal{H}^{\otimes n}
$$

with scalar product denoted by $\langle\cdot \mid \cdot\rangle_{T}$ is a Hilbert space.

We study the von Neumann algebra $\Gamma_{T}(\mathcal{H})$ generated by the operators

$$
\omega(f)=a(f)+a^{+}(f), f \in \mathcal{H}_{R} .
$$

The choice of the relations $(\star)$ was made since several examples of such structures were investigated in the literature. The case $T(f \otimes g)=(g \otimes f)$ is known as the canonical commutation relations (CCR). The case $T(f \otimes g)=-(g \otimes f)$ is known as the canonical anti-commutation relations (CAR). More generally for $T(f \otimes g)=q(g \otimes f)$, where $q \in$ $[-1,1]$ we use the name $q$-canonical commutation relations $(q$-CCR). The case $T=0$ plays a fundamental role. In [Vo] Voiculescu proved that $\Gamma_{0}(\mathcal{H})=V N\left(\mathbf{F}_{N}\right)$, where $\mathbf{F}_{N}$ denotes the free group with $N=\operatorname{dim} \mathcal{H}$ generators. Other examples as well as those mentioned above were studied in a series of papers (see e.g. [JSW], [BSp], [BKSp], [PW] and references therein).

For our needs we will use the following assumptions:

I. $T=T^{*},\|T\|=q<1$,

II. $T$ satisfies the braid relation,

III. Under the identification $\overline{\mathcal{H}}_{R} \simeq \mathcal{H}_{R}$ we have $T=\bar{T}$ on $\mathcal{H}_{R}$.

If we choose $\left\{e_{i}\right\}$ to be an orthonormal basis of $\mathcal{H}_{R}$ and define matrix coefficients of $T$ by the formula $\left\langle T\left(e_{a} \otimes e_{b}\right) \mid e_{c} \otimes e_{d}\right\rangle=t_{a b}^{c d}$ then the relations $(\star)$ have the form

$$
a_{i} a_{j}^{+}=\sum_{r, s} t_{j s}^{i r} a_{r}^{+} a_{s}+\delta_{i j} I d
$$

and the equation $T=\bar{T}$ on $\mathcal{H}_{R}$ can be rewritten as $t_{j s}^{i r}=t_{i j}^{r s}$.

Under assumptions I, II, III the algebra $\Gamma_{T}(\mathcal{H})$ can be continuously embedded into the representation space. The map $D: \Gamma_{T}(\mathcal{H}) \rightarrow \mathcal{F}_{\mathcal{T}}(\mathcal{H})$ defined as $D(X)=X \Omega$ gives the embedding. The operator $\psi\left(f_{1} \otimes \ldots \otimes f_{n}\right)$ which corresponds to a vector $f_{1} \otimes \ldots \otimes f_{n}$ is called the Wick product of the operators $\omega\left(f_{1}\right), \ldots, \omega\left(f_{n}\right)$. Such an element of $\Gamma_{T}(\mathcal{H})$ 
exists and is unique. In $[\mathrm{K}]$ an explicit formula for the Wick product was given. It provides a useful tool for studying $\Gamma_{T}(\mathcal{H})$ (see $[\mathrm{K}]$ and $[\mathrm{Nu}]$ ).

In $[\mathrm{K}]$ we proved that if the number of generators is infinite then for $T$ such that $\|T\|=q<1$ the algebra $\Gamma_{T}(\mathcal{H})$ is a type II factor. In this paper we show that the result remains true if $\operatorname{dim} \mathcal{H}$ is greater than some finite natural number $N(q)$. Recently E. Ricard $[R]$ proved factoriality without restriction on the dimension. We generalize and present another method of the proof of the result of Sniady.

1. Notations and known theorems. This section is based on [BSp] and [Bo]. Unless otherwise stated we assume that $T \in B\left(\mathcal{H}_{R} \otimes \mathcal{H}_{R}\right)$ is a selfadjoint, strict contraction $(\|T\|=q<1)$ which satisfies the braid relation.

Definition 1. For fixed $m$ define a function $\varphi: S_{m} \rightarrow B(\mathcal{F}(\mathcal{H}))$ by quasi multiplicative extension of

$$
\varphi(e)=1, \quad \varphi\left(\pi_{i}\right)=T_{i}, \quad i \leq m-1,
$$

where

$$
T_{i}\left(f_{1} \otimes \ldots \otimes f_{s}\right)=f_{1} \otimes \ldots \otimes f_{i-1} \otimes T\left(f_{i} \otimes f_{i+1}\right) \otimes \ldots \otimes f_{s} .
$$

This means that for a reduced word $\sigma=\pi_{i_{1}} \cdots \pi_{i_{k}}$ we put $\varphi(\sigma)=T_{i_{1}} \cdots T_{i_{k}}$.

Definition 2. Fix the operator $T$ and define $P^{(n)}=\sum_{\sigma \in S_{n}} \varphi(\sigma)$.

According to the main theorem in $[\mathrm{BSp}] P^{(n)}$ is nonnegative for every $n$ (even strictly positive in case $\|T\|<1$ ) and therefore we can introduce a new scalar product on $\mathcal{H}^{\otimes n}$

$$
\langle\xi \mid \eta\rangle_{T}=\left\langle P^{(n)} \xi \mid \eta\right\rangle
$$

The $T$-Fock space $\mathcal{F}_{\mathcal{T}}(\mathcal{H})$ is the completion of the Fock space $\mathcal{F}^{\text {fin }}(\mathcal{H})=\mathbf{C} \Omega \oplus \bigoplus_{n=1}^{\infty} \mathcal{H}^{\otimes n}$ with respect to the scalar product mentioned above.

Definition 3. For $f \in \mathcal{H}_{R}$ we define the left and right creation operators $a_{l}^{+}(f), a_{r}^{+}(f)$ as

$$
a_{l}^{+}(f) f_{1} \otimes \ldots \otimes f_{n}=f \otimes f_{1} \otimes \ldots \otimes f_{n} \quad \text { and } \quad a_{r}^{+}(f) f_{1} \otimes \ldots \otimes f_{n}=f_{1} \otimes \ldots \otimes f_{n} \otimes f
$$

and the right and left annihilation operators $a_{l}(f), a_{r}(f)$ as their adjoints on the $T$-Fock space.

By $[\mathrm{K}]$ the operators $a_{l}^{+}(f)$ and $a_{r}^{+}(f)$ are bounded and their adjoints are well defined. Explicit formulas for annihilation operators on $\mathcal{H}^{\otimes n}$ are the following

$$
a_{l}(f)=l_{l}(f)\left(1+T_{1}+T_{1} T_{2}+\ldots+T_{1} T_{2} T_{3} \cdots T_{n-1}\right)
$$

and

$$
a_{r}(f)=l_{r}(f)\left(1+T_{n-1}+T_{n-1} T_{n-2}+\ldots+T_{n-1} T_{n-2} \cdots T_{1}\right),
$$

where $l_{l, r}(f) \Omega=0$,

$$
\begin{aligned}
& l_{l}(f)\left(f_{1} \otimes \ldots \otimes f_{n}\right)=\left\langle f_{1} \mid f\right\rangle f_{2} \otimes \ldots \otimes f_{n}, \\
& l_{r}(f)\left(f_{1} \otimes \ldots \otimes f_{n}\right)=\left\langle f_{n} \mid f\right\rangle f_{1} \otimes \ldots \otimes f_{n-1} .
\end{aligned}
$$

Definition 4. Let $\Gamma_{T}(\mathcal{H})$ be the von Neumann algebra generated by the selfadjoint operators $\omega_{l}(f)=a_{l}(f)+a_{l}^{+}(f), f \in \mathcal{H}_{R}$ on $\mathcal{F}_{T}(\mathcal{H})$. 
The vector $\Omega$ is separating and cyclic for $\Gamma_{T}(\mathcal{H})$. Moreover the functional $\tau_{T}(X)=$ $\langle X \Omega \mid \Omega\rangle_{T}$ is a faithful normal trace. The map $D: \Gamma_{T}(\mathcal{H}) \rightarrow \mathcal{F}(\mathcal{H})$ defined as $D(X)=$ $X(\Omega)$ is a continuous embedding of $\Gamma_{T}(\mathcal{H})$ into $\mathcal{F}_{\mathcal{T}}(\mathcal{H})$.

Notation 5. For $\xi \in \mathcal{F}(\mathcal{H})$ we denote by $\psi(\xi)$ the unique element of $\Gamma_{T}(\mathcal{H})$ such that $\psi(\xi) \Omega=\xi$.

2. The result. Let $e_{1}, e_{2}, \ldots$ be an orthonormal basis of $\mathcal{H}_{R}$. Let

$$
\omega_{l}(f)=a_{l}(f)+a_{l}^{+}(f) \text { and } \omega_{r}(f)=a_{r}(f)+a_{r}^{+}(f) .
$$

Notice that $\omega_{r}\left(e_{i}\right) \in\left(\Gamma_{T}(\mathcal{H})\right)^{\prime}$. Take

$$
M=\sum_{i=1}^{d}\left(\omega_{l}\left(e_{i}\right)-\omega_{r}\left(e_{i}\right)\right)^{2},
$$

where $d$ is a natural number with $d \leq \operatorname{dim} \mathcal{H}$. This operator is positive and selfadjoint. We will show that

$$
\operatorname{Ker}(M)=\mathbf{C} \Omega .
$$

THEOREM 6. There exists $d_{0}$ such that for $d \geq d_{0}$ operator $M$ is strictly positive on $\mathcal{F}_{T}(\mathcal{H}) \backslash \mathbf{C} \Omega$.

To prove the theorem we need some estimates.

LEMMA 7. Fix $f \in \mathcal{H}_{R}^{\otimes n}$.

$$
\begin{gathered}
\left\|L_{n+1}\left(T_{n} \cdots T_{2} T_{1}\right) D_{j}(f)\right\|_{T} \leq q^{n}\|f\|_{T}, \\
\left\|L_{1}\left(T_{2} T_{3} \cdots T_{n+1}\right) D_{j}(f)\right\|_{T} \leq q^{n}\|f\|_{T},
\end{gathered}
$$

b)

where $D_{j}(f)=e_{j} \otimes f \otimes e_{j}, L_{n+1}\left(f_{1} \otimes \cdots \otimes f_{n+2}\right)=\left\langle f_{n+1} \mid f_{n+2}\right\rangle f_{1} \otimes \ldots \otimes f_{n}$ and $L_{1}\left(f_{1} \otimes \cdots \otimes f_{n+2}\right)=\left\langle f_{1} \mid f_{2}\right\rangle f_{3} \otimes f_{4} \otimes \cdots \otimes f_{n+2}$.

Proof. Notice that for arbitrary $\sigma \in S_{n}$ we have

$$
\begin{aligned}
L_{n+1}\left(T_{n} \cdots T_{2} T_{1}\right) D_{j} \varphi(\sigma) & =L_{n+1} \varphi(\rho \times I d) \varphi(I d \times \sigma \times I d) D_{j} \\
& =L_{n+1}(\varphi(\rho) \otimes I d)(\varphi(I d \times \sigma) \otimes I d) D_{j},
\end{aligned}
$$

where $\rho=\pi_{n} \cdots \pi_{1}$. Further one can verify that $\operatorname{Inv}(\rho)+\operatorname{Inv}(\operatorname{Id} \times \sigma)=\operatorname{Inv}(\rho(\operatorname{Id} \times \sigma))$. Furthermore $\rho(\operatorname{Id} \times \sigma)=(\sigma \times I d) \rho$ and also $\operatorname{Inv}(\sigma \times I d)+\operatorname{Inv}(\rho)=\operatorname{Inv}((\sigma \times I d) \rho)$. This implies that $\varphi(\rho) \varphi(I d \times \sigma)=\varphi(\rho(I d \times \sigma))=\varphi((\sigma \times I d) \rho)=\varphi(\sigma \times I d) \varphi(\rho)$ and finally gives

$$
\begin{aligned}
L_{n+1}\left(T_{n} \cdots T_{1}\right) D_{j} \varphi(\sigma) & =L_{n+1}(\varphi(\sigma \times I d) \otimes I d)(\varphi(\rho) \otimes I d) D_{j} \\
& =\varphi(\sigma) L_{n+1}(\varphi(\rho) \otimes I d) D_{j} \\
& =\varphi(\sigma) L_{n+1}\left(T_{n} \cdots T_{2} T_{1}\right) D_{j} .
\end{aligned}
$$

This implies that operator $L_{n+1}\left(T_{n} \cdots T_{2} T_{1}\right) D_{j}$ commutes with $P^{(n)}=\sum \varphi(\sigma)$ and therefore its norm is the same as on $\mathcal{H}^{\otimes n}$ with the usual scalar product.

The operator $L_{n+1}: \mathcal{H}^{\otimes(n+1)} \otimes e_{j} \rightarrow \mathcal{H}^{\otimes n}$ is a contraction, $D_{j}$ is an isometry and

$$
\left\|\left(T_{n} \cdots T_{2} T_{1}\right)\right\| \leq q^{n}
$$


LEMMA 8. The following estimations hold for $f \in \mathcal{F}_{T}(\mathcal{H})$ :

(a) $\left\|\sum_{i=1}^{d} e_{i} \otimes f \otimes e_{i}\right\|_{T}^{2} \leq d C_{q}^{2}\|f\|_{T}^{2}$

(b) $\left\|\sum_{i=1}^{d} a_{l}\left(e_{i}\right) a_{r}\left(e_{i}\right) f\right\|_{T}^{2}=\left\|\sum_{i=1}^{d} a_{r}\left(e_{i}\right) a_{l}\left(e_{i}\right) f\right\|_{T}^{2} \leq d C_{q}^{2}\|f\|_{T}^{2}$,

(c) $\left\|\sum_{i=1}^{d} e_{i} \otimes a_{r}\left(e_{i}\right) f\right\|_{T}^{2} \leq d C_{q}^{2}\|f\|_{T}^{2}$,

(d) $\left\|\sum_{i=1}^{d} a_{l}\left(e_{i}\right) f \otimes e_{i}\right\|_{T}^{2} \leq d C_{q}^{2}\|f\|_{T}^{2}$,

(e) $\left\|\sum_{i=1}^{d} a_{l}\left(e_{i}\right) a_{l}\left(e_{i}\right) f\right\|_{T}^{2} \leq d C_{q}^{2}\|f\|_{T}^{2}$

(f) $\left\|\sum_{i=1}^{d} a_{r}\left(e_{i}\right) a_{r}\left(e_{i}\right) f\right\|_{T}^{2} \leq d C_{q}^{2}\|f\|_{T}^{2}$,

(g) $\left\|\sum_{i=1}^{d} a_{l, r}^{+}\left(e_{i}\right) a_{l, r}\left(e_{i}\right) f\right\|_{T}^{2} \leq d C_{q}^{2}\|f\|_{T}^{2}$,

(h) $\left\|\sum_{i=1}^{d} \sum_{k, s} t_{i s}^{i k} e_{k} \otimes a_{l}\left(e_{s}\right) f\right\|_{T}^{2} \leq d q^{2} C_{q}^{2}\|f\|_{T}^{2}$.

Proof. Let $f=\sum f_{n}$, where $f_{n} \in \mathcal{H}^{\otimes n}$. Observe that $e_{i} \otimes f_{n} \otimes e_{i} \in \mathcal{H}^{\otimes n+2}$. Therefore for fixed $i$ we have

$$
\left\langle e_{i} \otimes f_{n} \otimes e_{i} \mid e_{i} \otimes f_{m} \otimes e_{i}\right\rangle_{T}=0 \text { if } n \neq m .
$$

It is known from [BSp] that there exists some constant $C_{q}$ (it can be explicitly calculated) such that for arbitrary $k<m$

$$
P_{T}^{(m)} \leq C_{q}\left(P_{T}^{(k)} \otimes P_{T}^{(m-k)}\right)
$$

This yields

$$
\begin{aligned}
\left\|\sum_{i=1}^{d} e_{i} \otimes f \otimes e_{i}\right\|_{T}^{2} & =\sum_{n=0}^{\infty}\left\|\sum_{i=1}^{d} e_{i} \otimes f_{n} \otimes e_{i}\right\|_{T}^{2} \\
& =\sum_{n=0}^{\infty}\left\langle\sum_{i=1}^{d} P_{T}^{(n+2)}\left(e_{i} \otimes f_{n} \otimes e_{i}\right) \mid \sum_{i=1}^{d} e_{i} \otimes f_{n} \otimes e_{i}\right\rangle \\
& \leq \sum_{n=0}^{\infty} C_{q}^{2}\left\langle\sum_{i=1}^{d}\left(I \otimes P_{T}^{(n)} \otimes I\right)\left(e_{i} \otimes f_{n} \otimes e_{i}\right) \mid \sum_{i=1}^{d} e_{i} \otimes f_{n} \otimes e_{i}\right\rangle .
\end{aligned}
$$

Since $\left\langle\left(I \otimes P_{T}^{(n)} \otimes I\right)\left(e_{i} \otimes f_{n} \otimes e_{i}\right) \mid e_{j} \otimes f_{n} \otimes e_{j}\right\rangle=0$ for $i \neq j$ the last expression is equal 
to

$$
\begin{aligned}
& \sum_{n=0}^{\infty} \sum_{i=1}^{d} C_{q}^{2}\left\langle\left(I \otimes P_{T}^{(n)} \otimes I\right)\left(e_{i} \otimes f_{n} \otimes e_{i}\right) \mid e_{i} \otimes f_{n} \otimes e_{i}\right\rangle \\
&=C_{q}^{2} \sum_{n=0}^{\infty} d\left\langle P_{T}^{(n)} f_{n} \mid f_{n}\right\rangle=d C_{q}^{2} \sum_{n=0}^{\infty}\left\|f_{n}\right\|_{T}^{2}=d C_{q}^{2}\|f\|_{T}^{2} .
\end{aligned}
$$

This gives (a).

For (b) define $B_{i} f=a_{r}\left(e_{i}\right) a_{l}\left(e_{i}\right) f$. By taking the adjoints on both sides we obtain $B_{i}^{*} f=a_{l}^{+}\left(e_{i}\right) a_{r}^{+}\left(e_{i}\right) f$. Further

$$
\left(\sum_{i=1}^{d} a_{r}\left(e_{i}\right) a_{l}\left(e_{i}\right)\right)^{*} f=\sum_{i=1}^{d} a_{l}^{+}\left(e_{i}\right) a_{r}^{+}\left(e_{i}\right) f=\sum_{i=1}^{d} e_{i} \otimes f \otimes e_{i} .
$$

From (a) we have that $\left\|\sum_{i=1}^{d} a_{l}^{+}\left(e_{i}\right) a_{r}^{+}\left(e_{i}\right)\right\|_{\infty} \leq \sqrt{d} C_{q}$, which implies

$$
\left\|\sum_{i=1}^{d} a_{l}\left(e_{i}\right) a_{r}\left(e_{i}\right)\right\|_{\infty} \leq \sqrt{d} C_{q}
$$

To prove (c), as in (a) we write

$$
\begin{aligned}
\left\|\sum_{i=1}^{d} e_{i} \otimes a_{r}\left(e_{i}\right) f\right\|_{T}^{2} & =\sum_{n=0}^{\infty}\left\|\sum_{i=1}^{d} e_{i} \otimes a_{r}\left(e_{i}\right) f_{n}\right\|_{T}^{2} \\
& =\sum_{n=0}^{\infty}\left\langle\sum_{i=1}^{d} P_{T}^{(n)}\left(e_{i} \otimes a_{r}\left(e_{i}\right) f_{n}\right) \mid \sum_{i=1}^{d} e_{i} \otimes a_{r}\left(e_{i}\right) f_{n}\right\rangle \\
& \leq \sum_{n=0}^{\infty} C_{q}\left\langle\sum_{i=1}^{d}\left(I \otimes P_{T}^{(n-1)}\right)\left(e_{i} \otimes a_{r}\left(e_{i}\right) f_{n}\right) \mid \sum_{i=1}^{d} e_{i} \otimes a_{r}\left(e_{i}\right) f_{n}\right\rangle \\
& =\sum_{n=0}^{\infty} \sum_{i=1}^{d} C_{q}\left\langle\left(I \otimes P_{T}^{(n-1)}\right)\left(e_{i} \otimes a_{r}\left(e_{i}\right) f_{n}\right) \mid e_{i} \otimes a_{r}\left(e_{i}\right) f_{n}\right\rangle \\
& =C_{q} \sum_{n=0}^{\infty} \sum_{i=1}^{d}\left\langle P_{T}^{(n-1)}\left(a_{r}\left(e_{i}\right) f_{n}\right) \mid a_{r}\left(e_{i}\right) f_{n}\right\rangle \\
& =C_{q} \sum_{n=0}^{\infty} \sum_{i=1}^{d}\left\|a_{r}\left(e_{i}\right) f_{n}\right\|_{T}^{2} \leq C_{q} \sum_{n=0}^{\infty} \sum_{i=1}^{d} C_{q}\left\|f_{n}\right\|_{T}^{2}=C_{q}^{2} d\|f\|_{T}^{2} .
\end{aligned}
$$

In the last inequality we used an estimate of the norm of $a_{r}\left(e_{i}\right)$, i.e. $\left\|a_{r}\left(e_{i}\right)\right\|_{\infty} \leq \sqrt{C_{q}}$.

For $(\mathrm{d})$ it is obvious that $e_{i} \otimes a_{r}\left(e_{i}\right) f=a_{l}^{+}\left(e_{i}\right) a_{r}\left(e_{i}\right) f$. Also $a_{l}\left(e_{i}\right) f \otimes e_{i}=a_{r}^{+}\left(e_{i}\right) a_{l}\left(e_{i}\right) f$ $=\left(a_{l}^{+}\left(e_{i}\right) a_{r}\left(e_{i}\right)\right)^{*} f$. Now (d) can be derived from (c). Analogously we can prove (e), (f), (g). Finally for (h) and for $f=\sum f_{n}$, where $f_{n} \in \mathcal{H}^{\otimes n}$ we have

$$
\begin{aligned}
\sup _{\|f\|=1}\left\|\sum_{i=1}^{d} \sum_{k, s} t_{i s}^{i k} e_{k} \otimes a_{l}\left(e_{s}\right) f\right\|_{T}^{2} & =\sup _{\|f\|=1}\left\|\sum_{k, s} \sum_{i=1}^{d} t_{i s}^{i k} e_{k} \otimes a_{l}\left(e_{s}\right) f\right\|_{T}^{2} \\
& =\sup _{\|f\|=1} \sum_{n=0}^{\infty}\left\|\sum_{k, s} \sum_{i=1}^{d} t_{i s}^{i k} e_{k} \otimes a_{l}\left(e_{s}\right) f_{n}\right\|_{T}^{2} \leq C_{q} \sup _{\|f\|=1} \sum_{n=0}^{\infty} \sum_{k}\left\|\sum_{s} \sum_{i=1}^{d} t_{i s}^{i k} a_{l}\left(e_{s}\right) f_{n}\right\|_{T}^{2}
\end{aligned}
$$




$$
\begin{aligned}
& =C_{q} \sum_{k}\left\|\sum_{s} \sum_{i=1}^{d} t_{i s}^{i k} a_{l}\left(e_{s}\right)\right\|_{\infty}^{2}=C_{q} \sum_{k}\left\|\sum_{s} \sum_{i=1}^{d} \overline{t_{i s}^{i k}} a_{l}^{+}\left(e_{s}\right)\right\|_{\infty}^{2} \\
& =C_{q} \sum_{k} \sup _{\|f\|=1} \sum_{n=0}^{\infty}\left\|\sum_{s} \sum_{i=1}^{d} \overline{t_{i s}^{i k}} e_{s} \otimes f_{n}\right\|_{T}^{2} \leq C_{q}^{2} \sum_{n=0}^{\infty} \sum_{k, s}\left|\sum_{i=1}^{d} \overline{t_{i s}^{i k}}\right|^{2}\left\|f_{n}\right\|_{T}^{2} \\
& =C_{q}^{2} \sum_{k, s}\left|\sum_{i=1}^{d} t_{i s}^{i k}\right|^{2}=C_{q}^{2}\left\|T\left(\sum_{i=1}^{d} e_{i} \otimes e_{i}\right)\right\|^{2} \leq d q^{2} C_{q}^{2} .
\end{aligned}
$$

Now we can pass to the proof of our main theorem.

Proof of Theorem 6. We have

$$
\begin{aligned}
\sum_{i=1}^{d}\left(\omega_{l}\left(e_{i}\right)-\omega_{r}\left(e_{i}\right)\right)^{2} & =\sum_{i=1}^{d} a_{l}\left(e_{i}\right) a_{l}\left(e_{i}\right)+a_{l}^{+}\left(e_{i}\right) a_{l}^{+}\left(e_{i}\right)+a_{l}^{+}\left(e_{i}\right) a_{l}\left(e_{i}\right)+a_{l}\left(e_{i}\right) a_{l}^{+}\left(e_{i}\right) \\
& +\sum_{i=1}^{d} a_{r}\left(e_{i}\right) a_{r}\left(e_{i}\right)+a_{r}^{+}\left(e_{i}\right) a_{r}^{+}\left(e_{i}\right)+a_{r}^{+}\left(e_{i}\right) a_{r}\left(e_{i}\right)+a_{r}\left(e_{i}\right) a_{r}^{+}\left(e_{i}\right) \\
& -\sum_{i=1}^{d} a_{l}\left(e_{i}\right) a_{r}\left(e_{i}\right)+a_{l}^{+}\left(e_{i}\right) a_{r}^{+}\left(e_{i}\right)+a_{l}^{+}\left(e_{i}\right) a_{r}\left(e_{i}\right)+a_{l}\left(e_{i}\right) a_{r}^{+}\left(e_{i}\right) \\
& -\sum_{i=1}^{d} a_{r}\left(e_{i}\right) a_{l}\left(e_{i}\right)+a_{r}^{+}\left(e_{i}\right) a_{l}^{+}\left(e_{i}\right)+a_{r}^{+}\left(e_{i}\right) a_{l}\left(e_{i}\right)+a_{r}\left(e_{i}\right) a_{l}^{+}\left(e_{i}\right) .
\end{aligned}
$$

By the definition of $a_{r}\left(e_{i}\right)$ and $a_{l}\left(e_{i}\right)$ we have for $f \in \mathcal{H}^{\otimes n}$

$$
\begin{gathered}
a_{l}\left(e_{i}\right) a_{r}^{+}\left(e_{i}\right) f=a_{l}\left(e_{i}\right) f \otimes e_{i}+L_{1}\left(T_{2} T_{3} \cdots T_{n+1}\right) D_{j}(f), \\
a_{r}\left(e_{i}\right) a_{l}^{+}\left(e_{i}\right) f=e_{i} \otimes a_{l}\left(e_{i}\right) f+L_{n+1}\left(T_{n} \cdots T_{2} T_{1}\right) D_{j}(f), \\
a_{l}\left(e_{i}\right) a_{l}^{+}\left(e_{i}\right) f=f+\sum_{i=1}^{d} \sum_{k, s} t_{i s}^{i k} e_{k} \otimes a_{l}\left(e_{s}\right) f, \\
a_{r}\left(e_{i}\right) a_{r}^{+}\left(e_{i}\right) f=f+\sum_{i=1}^{d} \sum_{k, s} t_{i s}^{i k} a_{r}\left(e_{s}\right) f \otimes e_{k} .
\end{gathered}
$$

From Lemma 7 , for $f \in \mathcal{F}_{T} \backslash \mathbf{C} \Omega$

$$
\left\|L_{n+1}\left(T_{n} \cdots T_{2} T_{1}\right) D_{j}(f)\right\| \leq q\|f\|_{T} \quad \text { and } \quad\left\|L_{1}\left(T_{2} T_{3} \cdots T_{n+1}\right) D_{j}(f)\right\| \leq q\|f\|_{T} .
$$

Therefore $M=2 d I d+M_{1}-M_{2}+M_{3}$, where

$$
\left\|M_{1}\right\| \leq 2 \sqrt{d} C_{q} q, \quad\left\|M_{2}\right\| \leq 2 q d, \quad\left\|M_{3}\right\| \leq 14 C_{q} \sqrt{d} .
$$

This implies

$$
M \geq 2 d(1-q)-2 \sqrt{d} q C_{q}-14 \sqrt{d} C_{q} .
$$

It is easy to see that there exists $d_{0}$ such that the last expression is positive for arbitrary $d \geq d_{0}$.

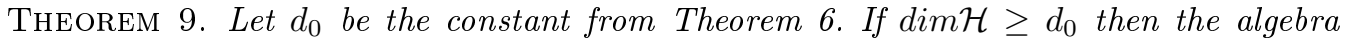
$\Gamma_{T}(\mathcal{H})$ is a factor. 
Proof. Notice that if $X \in \Gamma_{T}(\mathcal{H}) \cap \Gamma_{T}(\mathcal{H})^{\prime}$ then $X$ commutes with $\omega_{r}\left(e_{i}\right)$ and $\omega_{l}\left(e_{i}\right)$ for arbitrary $i$. Therefore $X \Omega \in \operatorname{Ker}(M)$. From Theorem 6 we derive that $\operatorname{Ker}(M)=\mathbf{C} \Omega$, hence $X=\alpha I d$ and $\Gamma_{T}(\mathcal{H})$ is a factor.

REMARK 10. The result can be generalized to the case of q-Araki-Woods algebras $([\mathrm{H}])$.

\section{References}

[BKSp] M. Bożejko, B. Kümmerer and R. Speicher, q-Gaussian processes: Non-commutative and classical aspects, Comm. Math. Phys. 185 (1997), 129-154.

[Bo] M. Bożejko, Ultracontractivity and strong Sobolev inequality for q-Ornstein-Uhlenbeck semigroup $(-1<q<1)$, Infin. Dimen. Anal. Quantum. Rel. Top. 2 (1999), 203-220.

[BSp] M. Bożejko and R. Speicher, Completely positive maps on Coxeter groups, deformed commutation relations and operator spaces, Math. Ann. 300 (1994), 97-120.

[BXu] M. Bożejko and Q. Xu, Factoriality and second quantization for von Neumann algebras of deformed commutation relations, preprint, Besançon, 2000.

[H] F. Hiai, q-deformed Araki-Woods factors, in: Operator Algebras and Mathematical Physics, Theta, Bucharest, 2003, 169-202.

[JSW] P. E. T. Jørgensen, L. M. Schmitt and R. F. Werner, Positive representations of general commutation relations allowing Wick ordering, J. Funct. Anal. 134 (1995), 3-99.

[K] I. Królak, Wick product for commutation relations connected with Yang-Baxter operators and new constructions of factors, Commun. Math. Phys. 210 (2000), 685-701.

[N] A. Nou, Non-injectivity of the q-deformed von Neumann algebras, Math. Ann. 330 (2004), 17-38.

[PW] W. Pusz and S. L. Woronowicz, Twisted second quantization, Rep. Math. Phys. 27 (1989), 231-257.

[R] E. Ricard, Factoriality of q-Gaussian algebras, Comm. Math. Phys. 257 (2005), 659665.

[Snia] P. Śniady, Factoriality of Bożejko-Speicher von Neumann algebras, Comm. Math. Phys. 246 (2004), 561-567.

[Vo] D. Voiculescu, Symmetries of some reduced free product of $C^{*}$ algebras, in: Operator Algebras and Ergodic Theory, Lecture Notes in Math. 1132, 1985, 556-588. 